\title{
Disinhibitory circuitry gates associative synaptic plasticity in olfactory cortex
}

\author{
Martha Canto-Bustos ${ }^{1,2}$, F. Kathryn Friason ${ }^{1,2}$, Constanza Bassi ${ }^{1}$ \\ and Anne-Marie M. Oswald ${ }^{1,2,3 *}$ \\ ${ }^{1}$ Department of Neuroscience, ${ }^{2}$ Center for the Neural Basis of Cognition, \\ University of Pittsburgh, Pittsburgh, PA, United States, 15213
}

\author{
Corresponding Author: \\ Anne-Marie M. Oswald \\ Associate Professor \\ Department of Neuroscience \\ University of Pittsburgh \\ A210 Langley Hall \\ Pittsburgh, PA, 15260 \\ ${ }^{3}$ Current Address \\ Department of Neurobiology \\ Neuroscience Institute \\ University of Chicago \\ Abbott Memorial Hall \\ 947 East $58^{\text {th }}$ St. MC0928 \\ Chicago, IL, USA, 60637 \\ amoswald@uchicago.edu
}




\section{Abstract}

2 Inhibitory microcircuits play an essential role in regulating cortical responses to sensory stimuli.

3 Interneurons that inhibit dendritic or somatic integration in pyramidal neurons act as

4 gatekeepers for neural activity, synaptic plasticity and the formation of sensory representations.

5 Conversely, interneurons that specifically inhibit other interneurons can open gates through

6 disinhibition. In the rodent piriform cortex, relief of dendritic inhibition permits long-term

7 potentiation (LTP) of the recurrent synapses between pyramidal neurons (PNs) thought to

8 underlie ensemble odor representations. We used an optogenetic approach to identify the

9 inhibitory interneurons and disinhibitory circuits that regulate LTP. We focused on three

10 prominent inhibitory neuron classes- somatostatin (SST), parvalbumin (PV), and vasoactive

11 intestinal polypeptide (VIP) interneurons. We find that LTP is gated by the inactivation SST or

12 PV interneurons or by activation of VIP interneurons. Further, activation of VIP interneurons

13 strongly inhibits putative SST-cells during LTP induction, but only weakly inhibit PV-

14 interneurons. Taken together, these findings suggest that VIP-interneurons mediate a

15 disinhibitory circuit that can regulate synaptic plasticity during olfactory processing. 
Throughout the cortex, the response properties of individual neurons as well as coordinated ensemble activity are refined by sensory experience. One underlying feature of experience-dependent plasticity is long-term changes in synaptic strength within cortical circuits.

30 While the mechanisms underlying excitatory synaptic plasticity have been extensively studied

31 (Abbott and Nelson 2000, Malenka and Bear 2004), less is known about the role cortical

32 circuitry plays in gating changes in synaptic strength. Inhibitory interneurons regulate both

33 dendritic integration and neural activity, two major factors in synaptic plasticity. Thus, inhibitory

34 circuits can play a key role in the enhancement of synaptic connections (Artinian and Lacaille

35 2018, Lucas and Clem 2018). In this study, we elucidate the inhibitory and disinhibitory circuit

36 motifs that gate synaptic plasticity at recurrent excitatory synapses in the olfactory cortex.

The anterior piriform cortex (APC) processes olfactory information, and performs both sensory and associative cortical functions. Located two synapses from the periphery; the APC is a primary cortical area representing odor inputs. Olfactory receptor neurons in the nose project

40 to Mitral and Tufted (M/T) neurons in the olfactory bulb (OB)(Mombaerts, Wang et al. 1996).

$41 \mathrm{M} / \mathrm{T}$ neurons then project directly to APC and synapse with pyramidal neurons (PNs)(Haberly

42 and Price 1977). Unlike other primary sensory cortices, APC lacks a topological representation

43 of odor identity. M/T axons project diffusely and randomly (Sosulski, Bloom et al. 2011, Igarashi,

44 leki et al. 2012) to activate distributed neural ensembles (Illig and Haberly 2003, Rennaker,

45 Chen et al. 2007, Stettler and Axel 2009). Distributed odor representations are further supported

46 by uniform intracortical excitatory connectivity across the APC (Franks, Russo et al. 2011). It is

47 postulated that odor-specific ensembles are constructed by strengthening excitatory synapses

48 between pyramidal neurons co-activated by odor components (Haberly 2001, Wilson and

49 Sullivan 2011). In support of this hypothesis, intracortical synapses between PNs are

50 strengthened following odor learning in vivo (Saar, Grossman et al. 2002) and through pairing of

51 afferent and intracortical stimulation in vitro (Kanter and Haberly 1993, Johenning, Beed et al.

52 2009). Hence, APC circuitry also supports early stage, associative odor processing. 
The APC is an ideal structure for investigating the circuit and synaptic plasticity

54 mechanisms that underlie sensory representations. PNs receive compartmentalized excitatory

55 inputs on their apical dendrites from two distinct fiber tracts. M/T cell afferents synapse distally,

56 while PN axons form a proximal intracortical fiber tract (Haberly and Price 1977, Haberly and

57 Price 1978). Co-activation of afferent and intracortical fiber tracts strengthens intracortical

58 synapses onto PNs through NMDA receptor (R) dependent, associative LTP (Kanter and

59 Haberly 1993). Further, NMDAR EPSPs and associative LTP induction are facilitated by

60 dendritic disinhibition by GABA receptor antagonists (Kanter and Haberly 1993, Kanter, Kapur

61 et al. 1996). While these studies suggest interplay between dendritic inhibition and disinhibition

62 gates LTP induction at intracortical synapses, the inhibitory circuitry involved has not been

63 identified.

64 Olfactory stimuli recruit both feedforward and recurrent inhibition onto PNs in APC (Poo

65 and Isaacson 2009, Poo and Isaacson 2011). Feedforward inhibition is comparatively weak and

66 diminishes with high frequency stimulation of the afferent pathway, whereas recurrent inhibition

67 is strong, and increases with stimulation through synaptic facilitation and PN recruitment

68 (Stokes and Isaacson 2010, Suzuki and Bekkers 2010a, Large, Vogler et al. 2016). Recurrent

69 inhibition is mediated by inhibitory interneurons that express somatostatin (SST-INs) or

70 parvalbumin (PV-INs) (Stokes and Isaacson 2010, Suzuki and Bekkers 2010a, Suzuki and

71 Bekkers 2010b, Large, Kunz et al. 2016). SST-INs inhibit PN apical dendrites proximal to the

72 soma, and are optimally located to regulate plasticity of intracortical synapses (Suzuki and

73 Bekkers 2010b, Large, Kunz et al. 2016). PV-INs regulate spike activity and could also impact

74 LTP through backpropagation (Johenning, Beed et al. 2009). A third class of interneurons-

75 vasoactive intestinal polypeptide interneurons (VIP-INs); inhibit SST and PV-INs and could

76 disinhibit pyramidal neurons (Lee, Kruglikov et al. 2013, Pfeffer, Xue et al. 2013, Karnani,

77 Jackson et al. 2016). VIP-INs are a prominent in APC (Suzuki and Bekkers 2010b) but their

78 inhibitory connections and function are unknown. We investigated the connectivity and 
79 functional roles of SST, PV and VIP-INs in APC. We find that activation of VIP-INs as well as

80 inactivation of SST-INs or PV-INs promote LTP of intracortical synapses. VIP-INs strongly inhibit

81 SST-INs during LTP induction, but only weakly inhibit PV-INs and PNs. Our finding suggests a

82 VIP->SST>-PN disinhibitory circuit gates associative LTP in APC.

83

84 Methods:

85 Mice: VIP-Cre (B6:Viptm1(cre)Zjh/J), SST-Cre (B6:Sst<tm2.1(cre)Zjh>/J) and PV-Cre mice 86 express cre-recombinase (Taniguchi, He et al. 2011). These mice were crossed with Ai32 mice

87 (B6;129S-Gt ROSA)26Sortm32 (CAG-COP4*H134R/EYFP)Hze/J) to express channelrhodopsin

88 (ChR2) or Ai35 mice (B6.129S-Gt(ROSA)26Sortm35.1(CAG- aop3/GFP)Hze/J) to express

89 archaerhodopsin (Arch) (Madisen, Mao et al. 2012). All mice are from Jackson Laboratory. All

90 animals were bred, handled and treated in manner that was evaluated and approved by the

91 Animal Care and Use Committee at the University of Pittsburgh IACUC, protocol \#17070877.

92 Slice preparation: APC brain slices were prepared from mice aged P19-35. The mice were

93 anesthetized with isoflurane and the brain was removed and immersed in ice cold oxygenated

$94\left(95 \% \mathrm{O}_{2}-5 \% \mathrm{CO}_{2}\right)$ ACSF (in mM: $125 \mathrm{NaCl}, 2.5 \mathrm{KCl}, 25 \mathrm{NaHCO}_{3}, 1.25 \mathrm{NaH}_{2} \mathrm{PO}_{4}, 1.0 \mathrm{MgCl}_{2}, 25$

95 Glucose, $2.5 \mathrm{CaCl}_{2}$ ) (all chemicals from Sigma, USA unless otherwise stated). Parasagittal

96 slices $(300 \mu \mathrm{m})$ were cut on a vibratome (Leica Biosystems) in ice-cold ACSF. The slices were

97 transferred to warm ACSF $\left(36^{\circ} \mathrm{C}\right)$ for $30 \mathrm{~min}$, then $20-22^{\circ} \mathrm{C}$ for 1 hour, and recorded at $25-28^{\circ} \mathrm{C}$.

98 Electrophysiology: Recordings were performed using a MultiClamp 700B amplifier (Molecular

99 Devices, Union City, CA). Data were low pass filtered (4 kHz) and digitized at $10 \mathrm{kHz}$ using an

100 ITC-18 (Instrutech) controlled by custom software (Recording Artist,

101 https://bitbucket.org/rgerkin/recording-artist) written in IgorPro (Wavemetrics). Recording

102 pipettes (4-10 M $\Omega$ ) were pulled from borosilicate glass (1.5 mm, outer diameter) on a

103 Flaming/Brown micropipette puller (Sutter Instruments). The series resistance (<20 M $\Omega$ ) was not 
104 corrected. For PSPs the intracellular solution consisted of (in mM) $130 \mathrm{~K}$-gluconate, $5 \mathrm{KCl}, 2$

$105 \mathrm{MgCl}_{2}$, 4 ATP-Mg, 0.3 GTP, 10 HEPES, and 10 phosphocreatine, $0.05 \%$ biocytin. For IPSC

106 recordings, Qx-314 was added to the K-gluconate internal (holding potential $0 \mathrm{mV}$ ) or Cs-Glu-

107 Qx solution was used (in mM, $130 \mathrm{Cs}-$ Gluconate, $5 \mathrm{KCl}, 2 \mathrm{MgCl}_{2}, 4 \mathrm{Mg}$-ATP, $0.3 \mathrm{GTP}, 10$

108 HEPES, 10 Phosphocreatine, 1 Qx-314, holding potential $+30 \mathrm{mV}$ ). Neurons were visualized

109 using infrared-differential interference contrast microscopy (IR-DIC, Olympus). For all recorded

110 neurons, subthreshold response properties were obtained using a series of hyperpolarizing and

111 depolarizing current steps (-50 pA to $50 \mathrm{pA}, 1 \mathrm{~s}$ duration). We used specific criteria for

112 identification of putative PV and SST interneurons based on intrinsic properties of identified PV

113 and SST neurons in APC (see (Large, Kunz et al. 2016, for details). Neural identity was

114 confirmed post hoc using intrinsic properties and biocytin fills.

115 LTP induction: Electrical stimulation was delivered using concentric bipolar electrodes (FHC).

116 The electrodes were placed in the LOT (L1a) and the L1b/L2 border. Stimuli (100 $\mu$ s pulse

117 width) were delivered through a stimulus isolation unit. Theta burst stimulation (TBS) of L1a

118 consisted of 10 bursts of 4 pulses $(100 \mathrm{~Hz})$ delivered at 250 ms intervals (Kanter and Haberly

119 1993). TBS stimulation intensity was set near spike threshold for the recorded neuron. L1b was

120 stimulated with a single weak pulse delivered between the $3^{\text {rd }}$ and $4^{\text {th }}$ pulse of each burst.

121 Stimulation intensity was $<30 \%$ of the maximum subthreshold EPSP $(\sim 1-6 \mathrm{mV})$. Pre and post

122 induction test pulses were delivered to L1b every $30 \mathrm{~s}$. Baseline was collected for $\sim 5$ min and

123 LTP was induced within 10 min of patching the neuron. Input resistance was monitored

124 throughout and neurons with deviations greater than $20 \%$ from baseline were excluded from

125 analysis ( $n=10$ neurons). We also monitored membrane potential throughout recording. Small

126 fluctuations $<5 \mathrm{mV}$ were offset with constant current to maintain driving force. However, neurons

127 with drift greater than $5 \mathrm{mV}$ or exhibiting unstable membrane potential fluctuations were

128 excluded from analysis $(n=8)$. 
129 Pharmacology: The GABA receptor antagonist, gabazine (GZ, $20 \mu \mathrm{M}$ in ACSF) was loaded

130 into a regular patch pipette and focally applied to L1b using a gentle positive pressure $(<1-5 \mathrm{~s}$

131 duration) see. Slices were oriented such that bath flowed from the soma to dendrite of the

132 pyramidal cell to maintain GZ in the region of the dendrite. Every effort was made to ensure

133 stability of GZ application including supplemental applications. In a subset of slices $(n=5)$ from

134 mice that expressed ChR2 in SST-INs, GZ application to L1b diminished optically evoked SST-

135 mediated inhibition onto pyramidal cells $(n=5)$. We verified that optically evoked IPSPs (Control:

$1366.3 \pm 0.69 \mathrm{mV}$ ) in these slices were stably diminished by Gabazine application throughout the

137 LTP protocol and did not differ between baseline (IPSP GZ: $0.89 \pm 0.27 \mathrm{mV}$ ) and post-induction

138 test pulses (IPSP GZ: $0.92 \pm 0.28, p>0.05$ WSR). In additional experiments, the NMDA receptor

139 antagonist, DL-2-Amino-5-phosphonopentanoic acid (DL-APV, $10 \mathrm{mM}$, Sigma-Aldrich) was

140 bath-applied as indicated in the main text.

141 Optogenetic Stimulation: Shutter controlled full field stimulation with blue (473 $\mathrm{nm}$ ) or green

142 (520 nm) light (Prior) was delivered through the epifluorescence pathway of the microscope

143 (Olympus) using a water-immersion objective (40x). Light intensity (5-10 mW) was adjusted to

144 induce spike responses (ChR2 activation) or spike suppression (Arch inactivation). Light pulse

145 duration varied by experiment as indicated in the main text.

146 EPSP Analysis: EPSPs were analyzed using custom software written in IgorPro (Wavemetrics).

147 Because we do not block inhibition during L1b test-pulses, we set narrow criteria to ensure that

148 only the excitatory portion of mixed EPSP-IPSPs was analyzed. Recurrent inhibition is

149 disynaptic and IPSPs are delayed with respect to excitation. Only EPSP onsets within 5 ms of

150 the stimulus artifact were analyzed. Across all conditions the average EPSP onset was $3.7 \pm$

$1510.41 \mathrm{~ms}$. EPSP peak amplitude was taken as the max amplitude within $5 \mathrm{~ms}$ of EPSP onset.

152 We also measured the slope of the rising phase of the EPSP from onset to $80 \%$ of peak

153 amplitude to further isolate the EPSP. Our core findings did not differ between peak and slope

154 measurements. 
155 Statistics: All data is presented as mean \pm SE. Statistical tests were performed using two tailed,

156 one or two-sample, paired or unpaired Student's t-test as appropriate. In cases of small sample

157 sizes non-parametric tests were used, including the Mann-Whitney U-test (MWU) for unpaired

158 data and the Wilcoxon Signed Ranks test (WSR) for paired data. For multiple comparisons we

159 used ANOVA with post hoc Tukey Test (ANOVA).

161 Results:

163 We investigated the roles of SST, PV and VIP-INs, in gating associative LTP at intracortical

164 excitatory synapses onto PNs. Afferent input to PNs arrives via L1a on distal L2 PN dendrites,

165 while the intracortical fiber tract $(\mathrm{L} 1 \mathrm{~b})$ is proximal (Fig 1A). L1a and L1b were easily identified

166 under IR-DIC and independently stimulated using bipolar electrodes. Associative LTP is induced

167 by pairing L1a and L1b stimulation using a theta burst stimulation (TBS) protocol (Kanter and

168 Haberly 1993) consistent with respiration coupled M/T spike frequencies (Kepecs, Uchida et al.

169 2007, Carey and Wachowiak 2011). Briefly, strong TBS of L1a was paired with weak, single

170 pulse stimulation of $L 1 b$ (see methods, Fig $1 A$ ). This $L 1 a+L 1 b$ pairing is hereafter denoted

171 induction. L1a-TBS evoked low $\mathrm{PN}$ firing rates ranging from 0-8 $\mathrm{Hz}$ and $\mathrm{L} 1 \mathrm{~b}$ EPSPs ranged from

172 1-6 mV. Pre and post induction, L1b stimulation was delivered every $30 \mathrm{~s}$. To avoid drift in

173 recording integrity, potentiation was quantified as the average L1b EPSP amplitude 25-30 min

174 following induction versus average baseline EPSP amplitude (5 min prior to induction).

175 However, in neurons where stability could be verified, potentiation typically lasted for the

176 duration of recording (45-60 min). Both raw and normalized (to baseline) EPSP amplitude and

177 rising slope were analyzed. PNs were excluded if input resistance or membrane potential varied

178 from baseline $( \pm 20 \%$ or $\pm 5 \mathrm{mV}$ respectively). 


\section{Disinhibition of PN dendrites promotes LTP}

With inhibition intact, the induction protocol did not induce LTP of L1b synapses. Neither

183 EPSP amplitude (in $\mathrm{mV}$, pre: $2.6 \pm 0.60$, post: $2.6 \pm 0.51$, p: 0.85 , paired t-test, $\mathrm{n}=9$ ) nor slope

184 (in $\mathrm{mV} / \mathrm{ms}$, pre: $0.64 \pm 0.20$, post: $0.61 \pm 0.18, \mathrm{p}: 0.27$, paired t-test, $\mathrm{n}=9$ ) significantly differed

185 from baseline (pre) at 30 min post induction (Fig 1B). Likewise, normalized EPSP amplitude

186 and area did not differ significantly from 1 (Amplitude: $1.0 \pm 0.071$, p: 0.65; Slope: $0.96 \pm 0.079$,

187 p: 0.65, one sample t-test, Fig 1C, D). However, when we focally applied the GABA $A_{A}$ receptor

188 antagonist, Gabazine (GZ, $20 \mu \mathrm{M})$, to L1b (schematic, Fig 1A), induction significantly enhanced

189 EPSP amplitude (mV, Pre: $1.3 \pm 0.32$, Post: $3.1 \pm 0.95, p<0.050$ WSR, n=6, Fig 1B) and slope

190 (mV/ms, Pre: $0.28 \pm 0.070$, Post: $0.52 \pm 0.12, p<0.050$ WSR, $n=6)$. Normalized EPSP amplitude

191 and slope were significantly greater than 1 (Amplitude: $2.3 \pm 0.35, p<0.05$ WSR; Slope: $2.0 \pm$

192 0.27, p<0.05 WSR, Fig 1C, D). Consistent application of dendritic Gabazine for the duration of

193 recording is difficult to maintain. In a some PNs, EPSP amplitudes were potentiated but

194 unstable, these were excluded from analysis $(n=5)$. This highlights the need for a more selective

195 and reliable optogenetic approach to investigating disinhibition.

Inactivation of SST-INs promotes LTP

We have previously shown that SST-interneurons strongly inhibit PN dendrites in L1B

198 (Large, Kunz et al. 2016). Although SST-INs typically receive weak direct L1a input (Suzuki and

199 Bekkers 2010a), SST-INs are recruited by strong TBS of L1a through the recruitment of

200 recurrent excitation (Fig 2A1,2). To investigate the influence of SST-INs on LTP induction,

201 Archaerhodopsin (Arch) was selectively expressed in SST-INs for optical inactivation. During

202 TBS, optical inactivation reduced SST-IN firing rate (FR) from $6.8 \pm 1.2 \mathrm{~Hz}$ to $1.8 \pm 0.4 \mathrm{~Hz}$

203 ( $p<0.05$, WSR test, Fig 2A2) and enhanced depolarization in PNs (Fig 2B). These findings

204 suggest that SST-interneurons mediate inhibition during TBS stimulation and could prevent

205 associative synaptic plasticity at L1B synapses. To test this, we optically inactivated SST-INs

206 solely during LTP induction (2 sec). EPSP amplitude and slope was significantly enhanced 30 
207 min post induction (Amplitude $(\mathrm{mV})$, Pre: $2.6 \pm 0.41$, Post: $4.3 \pm 0.76, \mathrm{p}: 0.0071$; Slope $(\mathrm{mV} / \mathrm{ms})$,

208 Pre: $0.55 \pm 0.14$, Post: $1.1 \pm 0.23$, p: 0.0048; $n=9$, paired t-test, Fig 2C1,2, E). Normalized

209 EPSP amplitude and slope were significantly greater than 1 (Amplitude: $1.7 \pm 0.16$, p: 0.00010;

210 Slope: $2.0 \pm 0.24$, p: 0.0033 , one sample t-test, Fig 2D). EPSP traces from representative PNs

211 pre and post induction are shown in Fig 2E. The normalized amplitude and slope averaged

212 across neurons over 30 min are shown in Fig 2F1,2. To confirm that associative synaptic

213 plasticity was NMDA-receptor dependent, we bath applied the NMDAR antagonist, DL-APV (10

$214 \mathrm{mM}$ ) to a second set of slices. Antagonism of NMDAR prevented LTP induction despite

215 inactivation of SST-INs. EPSP amplitude and slope $30 \mathrm{~min}$ post induction did not significantly

216 differ from baseline (Amplitude (mV): Pre: $4.7 \pm 0.81$, Post: $4.0 \pm 0.73, \mathrm{p}: 0.21$; Slope $(\mathrm{mV} / \mathrm{ms})$,

217 Pre: $0.83 \pm 0.19$, Post: $0.74 \pm 0.18$, p: 0.26; $n=10$, paired t-test, Fig 2C, black circles);

218 (normalized Amplitude: $0.88 \pm 0.13$, p: 0.38; Slope: $0.85 \pm 0.090$, p: 0.28, one sample t-test. Fig

219 2D,F black circles). These findings suggest that inhibition by SST-INs regulates NMDA-

220 dependent associative LTP at L1b intracortical synapses.

221 Inactivation of PV-INs promotes LTP

222 PV-INs strongly inhibit cortical neurons. We investigated whether optogenetic

223 inactivation of PV-INs expressing Arch also promotes associative LTP (Fig 3A1). PV-INs are

224 robustly activated during TBS (Fig 3A2) and light inactivation significantly decreased FR (Light

225 OFF: $7.2 \pm 2.5 \mathrm{~Hz}, \mathrm{ON}: 2.8 \pm 1.7 \mathrm{~Hz}, \mathrm{n}=5, \mathrm{p}<0.05$, WSR, Fig 3A2, right). In a number of PNs,

226 this enhanced EPSP summation (Fig 3B1) and/or FR consistent with somatic disinhibition. PV-

227 IN inactivation during induction promoted LTP of EPSP amplitude and slope (Fig 3C1,C2,

228 Amplitude (mV), Pre: $1.6 \pm 0.34$, Post: $2.4 \pm 0.40$, p: 0.00095 ; Slope (mV/ms): $0.38 \pm 0.10$, Post:

$2290.66 \pm 0.18, p: 0.016 ; n=9$, paired t-test). Normalized amplitude and slope were significantly $>1$

230 post induction (Amplitude: $1.6 \pm 0.16$, p: 0.0063; Slope: $1.8 \pm 0.15$, p: 0.00061 , one sample t-test

231 Fig 3C3, D). Antagonism of NMDAR prevented LTP induction mediated by inactivation of PV-

232 INs. EPSP amplitude and slope post induction did not significantly differ from baseline 
233 (Amplitude $(\mathrm{mV})$ : Pre: $3.3 \pm 0.45$, Post: $3.0 \pm 0.37$, p: 0.48; Slope (mV/ms), Pre: $0.44 \pm 0.082$,

234 Post: $0.52 \pm 0.10$, p: 0.74; n=8, paired t-test, Fig 3C, black circles); (normalized Amplitude:

$2350.92 \pm 0.076$, p: 0.65; Slope: $1.0 \pm 0.13$, p: 0.56, one sample t-test. Fig 3C,D black circles).

236 Thus, PV-INs can also regulate the induction of NMDA-dependent associative LTP at L1b

237 intracortical synapses.

238 Inhibition of interneurons by VIP-INs

239 If both SST-IN and PV-INs can inhibit LTP induction, the question remains as to which

240 circuits might provide a means for disinhibition and the promotion of LTP. In other cortices, VIP

241 interneurons inhibit SST-INs and PV-INs (Pfeffer, Xue et al. 2013, Pi, Hangya et al. 2013).

242 Although there are numerous VIP-INs in piriform cortex (Suzuki and Bekkers 2010b), their

243 targets have not been identified. We crossed VIP-cre mice with Ai32 mice to express

244 channelrhodopsin (ChR2) in VIP-INs. We then used light activation to drive single action

245 potentials in VIP-INs while recording potential postsynaptic targets. Typically, VIP-cre mice are

246 crossed with mice that express GFP in SST-INs (GIN-mice) or PV-INs (G42-mice). However,

247 these lines sparsely label SST and PV-INs in APC (Large, Kunz et al. 2016). Instead, we used

248 intrinsic properties to identify putative (p) SST-INs and pPV-INs according criteria determined

249 from recordings in genetically identified SST-INs and PV-INs in APC (Large, Kunz et al.

250 2016)(see methods). Interneurons that could not be confidently identified were excluded from

251 analysis.

252 VIP-INs were activated by brief blue light pulses $(5 \mathrm{~ms})$ and IPSCs were recorded in

253 voltage clamp with either Cs-Gluconate-Qx internal (IPSCs, $+30 \mathrm{mV}$ ) or K-Gluconate-Qx internal

254 (IPSCs, $0 \mathrm{mV}$ ). IPSC recordings were most reliable with Cs-Glu-Qx and the data presented (Fig

255 4) are from this condition. VIP-INs inhibited nearly all recorded pSST-INs (86\%), most pPV-INs

256 (90\%) and PNs (88\%). Although high connectivity with SST and PV cells was expected, this

257 was unexpected for PNs (Pfeffer, Xue et al. 2013). VIP-INs strongly inhibited pSST-INs (IPSC

258 amplitude: $303 \pm 57 \mathrm{pA}, \mathrm{n}=19$ ). However, despite a high probability of connection, VIP-INs very 
weakly inhibited pPV-INs (35 \pm 5.8 pA, n=9, p: 0.0008) and PNs $(61 \pm 14$ pA, p: $0.001 \mathrm{n}=14$,

260 ANOVA, Fig 4A1). Qualitatively similar results were found with K-Glu-Qx, however IPSC

261 amplitude and connection probability were considerably lower (\% connected, amplitude, \#

262 connected: pSST: $73 \%, 47 \pm 14$ pA, n=19; pPV: 73\%, $31 \pm 7.6$ pA, n=11; PN: 29\%, $21 \pm 7.0$ pA,

$263 n=4)$. ms of suprathreshold depolarizations by current steps. For this reason, long light pulses (2 s)

266 during LTP induction would likely result in significant adaptation of VIP-INs spike responses.

267 Instead, we drove VIP-INs with shorter light pulses $(100 \mathrm{~ms})$ delivered at theta frequency (10

268 pulses, Fig 4A2). This pulsed-light stimulation evoked strong and relatively sustained IPSCs in

269 pSST-INs (Fig 4B2 top). Overall, IPSC strength decreased by only $\sim 30 \%$ by the $5^{\text {th }}$ pulse in all

270 cell types then stabilized (SST: $31 \pm 10 \%$, p: 0.002; PV: 31 5\%, p: 0.06; PC: $28 \pm 12 \%, p: 0.02$,

271 paired t-test, Fig 4B2).

272 To investigate how VIP-IN mediated inhibition could influence action potential activity we 273 depolarized target neurons to near or suprathreshold membrane potentials in current clamp. In

274 pSST-INs activation of VIP-INs evoked strong IPSPs (2.02 $\pm 0.38 \mathrm{mV}, \mathrm{n}=18$, Fig 4C1). These

275 IPSPs were sufficient to delete or delay spikes by an average of $77 \pm 26 \mathrm{~ms}$ ( $\mathrm{p}: 0.016, \mathrm{n}=11$,

276 paired t-test) compared to non-light trials (Fig 4C1,3 left). Finally, pSST-IN spike responses

277 evoked by TBS were significantly decreased by pulsed activation of VIP-INs compared to non-

278 light trials (OFF: $5.0 \pm 0.6 \mathrm{~Hz}, \mathrm{ON}: 3.5 \pm 0.5, \mathrm{p}: 0.002, \mathrm{n}=11$, paired t-test, Fig 4C). We could not

279 record IPSPs in PNs $(n=7)$ or pPV-INs $(n=4)$ likely due to a combination of low input resistance

280 and weak VIP-mediated IPSCs. Taken together, these findings suggest that VIP-INs could

281 strongly influence pSST-INs during LTP induction but would have considerably less impact on

282 pPV-IN or PNs. 


\section{Activation of VIP-INs promotes LTP}

VIP-INs are numerous in APC and we find that VIP-INs inhibit pSST-INs. VIP-to-SST inhibition is a candidate circuit motif for dendritic disinhibition of PNs that could promote LTP. So, why isn't LTP induced without antagonism of inhibition? We recorded VIP-INs during TBS stimulation of L1a. We found that in contrast to SST-INs or PV-INs, VIP-INs were only weakly driven to fire action potentials by TBS $(0.7 \pm 0.6 \mathrm{~Hz}, \mathrm{n}=6$, Fig 5A2). This lack of VIP-IN

290 recruitment is consistent with the inability to induce LTP under control conditions (Fig 1B).

291 However, light activation of ChR2+ VIP-INs enhanced FR during TBS $(14 \pm 4.0 \mathrm{~Hz}, p<0.05$,

292 WSR test Fig 5A2). Activating VIP-INs also enhanced EPSP summation in PNs during TBS

293 (Fig 5B). Activation of VIP-INs during induction lead to robust LTP of EPSP amplitude and slope

294 (Fig 5C1,C2, amplitude (mV), Pre: $2.1 \pm 0.50$, Post: $3.4 \pm 0.48, \mathrm{p}<0.02$; slope (mV/ms) Pre: 0.47

$295 \pm 0.081$, Post: $0.76 \pm 0.072, p<0.02, n=7$, WSR). Normalized amplitude and slope were

296 significantly $>1$ post induction (Amplitude: $2.0 \pm 0.33, p<0.02$; Slope: $1.9 \pm 0.32, p<0.02$, WSR,

297 Fig 5C3). Antagonism of NMDARs prevented LTP induction mediated by activation of VIP-INs.

298 EPSP amplitude and slope post induction did not significantly differ from baseline (Amplitude

299 (mV): Pre: $6.0 \pm 1.6$, Post: $5.5 \pm 1.5$, p>0.05; Slope $(\mathrm{mV} / \mathrm{ms})$, Pre: $1.2 \pm 0.23$, Post: $1.1 \pm 0.28$,

300 p: >0.05; n=5, WSR, Fig 5C,D black circles); (normalized Amplitude: $0.93 \pm 0.072, p>0.05$;

301 Slope: $0.99 \pm 0.17$, p >0.05, WSR. Fig 5C,D black circles). Altogether, these findings support a

302 VIP-SST-PN disinhibitory circuit that gates associative LTP at intracortical synapses.

\section{Discussion}

305 It has been hypothesized that odor ensembles are formed through associative plasticity of

306 recurrent excitatory synapses between co-activated pyramidal neurons (Haberly 2001, Wilson

307 and Sullivan 2011). These intracortical synapses are stronger in animals that have learned an

308 olfactory discrimination task (Saar, Grossman et al. 2002, Saar, Reuveni et al. 2012) and the

309 induction of associative LTP in vitro is occluded in animals that have learned tasks (Lebel, 
310 Grossman et al. 2001). This capacity for enhancement as well as highly recurrent excitation

311 (Poo and Isaacson 2011) necessitates strong inhibition to regulate neural activity (Luna and

312 Schoppa 2008, Poo and Isaacson 2011, Bolding and Franks 2018) and synaptic plasticity

313 (Kanter and Haberly 1993, Kanter, Kapur et al. 1996). Our present findings are consistent with a

314 VIP->SST->PN circuit that transiently disinhibits PN dendrites to promote synaptic plasticity

315 during odor learning while overall inhibition remains intact.

316 Associative LTP at intracortical synapses within APC is well-characterized (Stripling,

317 Patneau et al. 1988, Kanter and Haberly 1993, Poo and Isaacson 2007). TBS provides strong

318 afferent excitation that depolarizes apical PN dendrites and promotes NMDAR dependent

319 potentiation of co-activated intracortical synapses. However, strong TBS also recruits strong

320 inhibition and LTP is rarely induced in the absence of GABA $R$ antagonists (Kanter and Haberly

321 1993). We have reproduced previous findings that LTP is gated by dendritic application of

322 Gabazine (Kanter, Kapur et al. 1996, Kumar, Schiff et al. 2018). Further, we have shown that

323 dendritic application of Gabazine blocks inhibition mediated by SST-INs (Large et al., 2016). In

324 the present study, we demonstrate that inactivation of SST-INs at an opportune moment during

325 TBS, is sufficient to promote LTP. Thus, a circuit mechanism that inhibits SST-INs during LTP

326 induction could gate synaptic plasticity. Throughout the cortex, VIP-INs inhibit SST-INs and

327 potentially disinhibit PNs (Lee, Kruglikov et al. 2013, Pfeffer, Xue et al. 2013, Karnani, Jackson

328 et al. 2016). We show that VIP-INs strongly inhibit pSST-INs and weakly inhibit pPV-INs as well

329 as PNs. Moreover, optogenetic activation of VIP-INs decreases pSST-IN spike responses

330 during TBS. Finally, activation of VIP-INs specifically during TBS is sufficient gate associative

331 LTP. Based on these findings, we propose that a VIP->SST->PN disinhibitory circuit gates

332 intracortical synaptic plasticity in APC.

333 However, it is relevant to discuss three additional circuits that are potentially recruited

334 with VIP-IN activation. For example, VIP-INs could also gate plasticity through a VIP-PV-PN

335 disinhibitory circuit. Although optogenetic inactivation of PV-INs promotes LTP, we predict this 
336 circuit motif contributes minimally to associative LTP induction. VIP-INs very weakly inhibit

337 pPV-INs, which is likely insufficient to prevent PV-IN spiking during TBS. Conversely, SST-INs

338 strongly inhibit PV-INs (Pfeffer, Xue et al. 2013, Xu, Jeong et al. 2013, Large, Kunz et al. 2016)

339 and inactivation of SST-INs in APC increases pPV-IN activity (Sturgill and Isaacson, 2015).

340 Instead of disinhibiting the PN, a VIP-SST-PV circuit could transiently increase somatic PV-IN

341 inhibition to compensate for a loss of SST mediated inhibition at the dendrite and/or soma.

342 Finally, VIP-INs also inhibit PNs directly, possibly through basket like synapses on the soma

343 (Suzuki and Bekkers 2010a). As with VIP-PV synapses, this VIP-PN inhibitory circuit is also

344 weak and its role is unknown. Importantly, recruitment of these additional circuits does not

345 impair LTP induction with VIP-IN activation. Rather, the interplay of these circuits suggests a

346 shift in inhibition from the dendrite to soma that may stabilize neural responses while opening a

347 window for plasticity.

$348 \quad$ Our study complements a recent study in somatosensory cortex that used

349 chemogenetics to inhibit VIP-INs or SST-INs and prevent or promote LTP induction respectively

350 (Williams and Holtmaat 2019). Together, these two studies support a common VIP->SST->PN

351 disinhibitory circuit motif regulates synaptic plasticity across cortical areas. A benefit of the

352 present study is that by using optogenetics, we were ability to isolate the influence of

353 disinhibition to a brief time window ( 2 s) when afferent and intracortical pathways are co-

354 activated. This suggests that transient dendritic disinhibition is sufficient to gate the cascade of

355 intracellular mechanisms underlying synaptic enhancement. Thus, circuit mechanisms that

356 provide a means for VIP cells to be activated in conjunction with odor sampling could play a key

357 role in odor learning and ensemble formation.

358 Interestingly, VIP-INs are not recruited by pairing afferent and intracortical stimulation,

359 this may underlie the inability induce associative LTP with inhibition intact (Fig 1). At present, it

360 is not known how VIP-INs in APC are recruited. In other cortices, VIP-IN activity is enhanced by

361 arousal, locomotion, task engagement or reward (Lee, Kruglikov et al. 2013, Pi, Hangya et al. 
362 2013, Fu, Tucciarone et al. 2014, Jackson, Ayzenshtat et al. 2016), either through additional

363 excitatory drive from other cortical areas (Lee, Kruglikov et al. 2013, Williams and Holtmaat

364 2019) or through neuromodulation (Porter, Cauli et al. 1999, Lee, Hjerling-Leffler et al. 2010,

365 Alitto and Dan 2012, Kuchibhotla, Gill et al. 2017, Pronneke, Witte et al. 2019). Direct excitatory

366 inputs to VIP-INs would have to be well-timed with respect to sensory stimulation. Conversely,

367 neuromodulation could raise the excitability of VIP-INs such that afferent and intracortical drive

368 are sufficient to drive VIP-INs with appropriate timing. The APC receives input from higher

369 cortices including orbitofrontal cortex (Illig 2005) as well as neuromodulatory centers

370 (Zaborszky, Carlsen et al. 1986, Linster, Wyble et al. 1999). Both of these pathways have been

371 implicated in olfactory learning and plasticity (Patil, Linster et al. 1998, Patil and Hasselmo 1999,

372 Linster, Maloney et al. 2003, Li, Luxenberg et al. 2006, Chapuis and Wilson 2013, Cohen,

373 Wilson et al. 2015, Strauch and Manahan-Vaughan 2018). Future studies are needed to

374 ascertain the potential links between descending excitation and/or neuromodulation, the

375 recruitment of VIP-INs, and the gating of synaptic plasticity during olfactory processing.

376 Though many previous studies have focused on the influence of disinhibitory circuits on

377 FR or behavior, our study highlights a role for disinhibition in the dynamics that gate long-term

378 plasticity. Specifically, our findings suggest that the VIP-SST-PN circuit motif plays a central role

379 in gating synaptic plasticity in PN dendrites. Additional circuit mechanisms mediated through

380 VIP-INs that regulate PV-INs or PNs directly could serve a stabilizing role. These findings

381 demonstrate the challenge of the delineating roles for the individual circuit motifs that are nested

382 in complex neural networks. It remains to be determined if these mechanisms work in concert

383 during afferent and recurrent olfactory processing.

384

385

386 
Figure 1: Associative LTP of intracortical synapses is gated by dendritic disinhibition.

A) Schematic of APC circuit and stimulation paradigm. Strong (s) TBS of afferents (L1a) is paired with weak (w) single-pulses at the intracortical pathway (L1b). Gabazine (GZ) is focally

391 applied to the dendrite region in L1b (tan oval). B1) Representative average traces pre (gray)

392 and post induction in control (black) and with GZ(Tan). B2) Average EPSP amplitude (mV) pre

393 (-5 to $0 \mathrm{~min}$ ) and post induction (25-30 $\mathrm{min}$ ) with inhibition intact (black) or with dendritic GZ

394 (tan). B3) Average slope ( $\mathrm{mV} / \mathrm{ms}$ ) of the $20-80 \%$ rising phase of the EPSP pre and post

395 induction. C1) Time course of normalized EPSP amplitude pre and post induction (grey box,

$396 \mathrm{t}=0$ ) in control (black) and with dendritic Gabazine (tan). C2) Time course of normalized EPSP

397 slope, colors as in C2. C3) Normalized EPSP amplitude, and slope 30 min post induction in

398 control (black) and with GZ (tan).

Figure 2: SST-interneuron inactivation promotes associative LTP. A1) Schematic: SST-

401 INs express Archaerhodopsin (Arch). A2) SST-IN spike responses during TBS in control (black)

402 and inactivated (green) conditions ( ${ }^{*} \mathrm{p}<0.05$, WSR test). B) PN responses for a single TBS

403 burst. Left : Inactivation of SST-INs enhanced PN depolarization (green vs. black trace).

404 Right: Depolarization during SST-IN inactivation (green) is reduced by APV (gray trace). C1)

405 Average EPSP amplitude $(\mathrm{mV})$ pre (-5 to $0 \mathrm{~min})$ and post induction (25-30 min) with SST-

406 inactivation (Magenta) or with SST-inactivation plus bath application of the NMDAR antagonist,

407 APV (black). C2) Average slope $(\mathrm{mV} / \mathrm{ms})$ of the $20-80 \%$ rising phase of the EPSP pre and post

408 induction, colors as in B2. C3) Normalized EPSP amplitude and slope following pairing with

409 SST-IN inactivation (magenta circles). LTP is blocked by bath application APV (black). D1)

410 Representative average traces pre (gray) and post induction in with SST-IN inactivation

411 (magenta) or inactivation plus APV (Black). D2) Time course of normalized EPSP amplitude pre

412 and post induction with inactivation of SST-INs (green box, $\mathrm{t}=0$ ) in control (magenta) and with

413 bath application of APV (black). D3) Time course of normalized EPSP slope, colors as in D2. 
414 Figure 3: PV-interneuron inactivation during induction promotes associative LTP. A1)

415 Schematic: PV-INs express Archaerhodopsin (Arch). A2) PV-IN spike responses during TBS in

416 control (black) and inactivated (green) conditions. B) Representative PN responses for a single

417 TBS for control (black) versus PV-IN inactivation (green). C1) Average EPSP amplitude (mV)

418 pre (-5 to $0 \mathrm{~min}$ ) and post induction (25-30 $\mathrm{min}$ ) with PV-inactivation (Blue) or with PV-

419 inactivation plus bath application of the NMDAR antagonist, APV (black). C2) Average slope

$420(\mathrm{mV} / \mathrm{ms})$ of the $20-80 \%$ rising phase of the EPSP pre and post induction, colors as in B2. C3)

421 Normalized EPSP amplitude and slope following pairing with PV-IN inactivation (blue circles) or

422 with inactivation plus bath application APV (black). D1) Representative average traces pre

423 (gray) and post induction in with PV-IN inactivation (blue) or inactivation plus APV (Black). D2)

424 Time course of normalized EPSP amplitude pre and post induction with inactivation of PV-INs

425 (green box, $t=0$ ) in control (blue) and with bath application of APV (black). D3) Time course of

426 normalized EPSP slope, colors as in D2.

428 Figure 4: Inhibition by VIP-interneurons in piriform cortex. A1) VIP-INs express ChR2.

429 Optically evoked IPSCs were recorded in putative(p) pSST-INs (magenta), pPV-INs (blue) and

430 PNs (black). A2) IPSCs recorded in response to ten light pulses (100 ms duration, $4 \mathrm{~Hz}$, same

431 neurons as in A1). B1) IPSC amplitudes were stronger in pSST-INs versus pPV-INs ( $\left.{ }^{*} p: 0.014\right)$

432 or PNs ( ${ }^{*} \mathrm{p}: 0.042$, ANOVA). B2) In pSST-INs, IPSC amplitude diminishes by the $5^{\text {th }}$ pulse of

433 theta stimulation ( ${ }^{* *} \mathrm{p}: 0.009$, paired t-test). C1) IPSCs from VIP-INs delay pSST-IN spike

434 responses during suprathreshold depolarization (4 overlaid traces) Left: Control, Right:

435 activation of VIP-INs (100 ms pulse, blue). Magenta trace: VIP-IN mediated IPSC during

436 subthreshold depolarization. C2) Interspike interval (ISI) was significantly increased during

437 optical activation of VIP-INs (blue circles, p: 0.016, paired t-test, $n=11$ ) compared to light off

438 trials (black circles). D1) Spike responses in pSST-INs during TBS in control (magenta trace) 
439 and during pulsed light (blue trace). D2) pSST-IN firing rates decreased during TBS on light

440 trials (blue) versus control (black circles, p:0.002, paired t-test, $n=11$ ).

442 Figure 5: VIP-interneuron activation promotes associative LTP. A1) Circuit schematic: VIP-

443 INs express ChR2 and were activated using theta pulsed light during $L 1 \mathrm{a}+\mathrm{L} 1 \mathrm{~b}$ pairing. A2) VIP-

444 IN responses during TBS without (black) and with light (blue). FRs increase during pairing with

445 light (blue circles, $p<0.05$, WSR, $n=7$ ). B) Top: Activation of VIP-INs enhanced PN

446 depolarization during TBS stimulation (blue vs. black trace). Bottom: PN depolarization during

447 VIP-IN activation (blue trace) is reduced by APV (gray trace). C1) Average EPSP amplitude

$448(\mathrm{mV})$ pre (-5 to $0 \mathrm{~min})$ and post induction (25-30 $\mathrm{min}$ ) with VIP-IN activation (green) or with VIP-

449 IN activation plus bath application of APV (black). C2) Average slope ( $\mathrm{mV} / \mathrm{ms})$ of the rising

450 phase of the EPSP pre and post induction, colors as in C1. C3) Normalized EPSP amplitude

451 and slope following pairing with VIP-IN activation (green circles) or with activation plus APV

452 (black). D1) Representative average traces pre (gray) and post induction with VIP-IN activation

453 (green) or activation plus APV (Black). D2) Time course of normalized EPSP amplitude pre and

454 post induction with activation of VIP-INs (blue box, $\mathrm{t}=0$ ) in control (green) and with APV (black).

455 D3) Time course of normalized EPSP slope, colors as in D2. 
466 Abbott, L. F. and S. B. Nelson (2000). "Synaptic plasticity: taming the beast." Nat Neurosci 3

467 Suppl: 1178-1183.

468 Alitto, H. J. and Y. Dan (2012). "Cell-type-specific modulation of neocortical activity by basal

469 forebrain input." Front Syst Neurosci 6: 79.

470 Artinian, J. and J. C. Lacaille (2018). "Disinhibition in learning and memory circuits: New

471 vistas for somatostatin interneurons and long-term synaptic plasticity." Brain Res Bull 141:

$472 \quad 20-26$.

473 Bathellier, B., T. W. Margrie and M. E. Larkum (2009). "Properties of piriform cortex

474 pyramidal cell dendrites: implications for olfactory circuit design." 」 Neurosci 29(40):

475 12641-12652.

476 Bolding, K. A. and K. M. Franks (2018). "Recurrent cortical circuits implement

477 concentration-invariant odor coding." Science 361(6407).

478 Carey, R. M. and M. Wachowiak (2011). "Effect of sniffing on the temporal structure of

479 mitral/tufted cell output from the olfactory bulb." \Neurosci 31(29): 10615-10626.

480 Chapuis, J. and D. A. Wilson (2013). "Cholinergic modulation of olfactory pattern

481 separation." Neurosci Lett 545: 50-53.

482 Cohen, Y., D. A. Wilson and E. Barkai (2015). "Differential modifications of synaptic weights

483 during odor rule learning: dynamics of interaction between the piriform cortex with lower

484 and higher brain areas." Cereb Cortex 25(1): 180-191.

485 Franks, K. M., M. J. Russo, D. L. Sosulski, A. A. Mulligan, S. A. Siegelbaum and R. Axel (2011).

486 "Recurrent circuitry dynamically shapes the activation of piriform cortex." Neuron 72(1):

$487 \quad 49-56$.

488 Fu, Y., J. M. Tucciarone, J. S. Espinosa, N. Sheng, D. P. Darcy, R. A. Nicoll, Z. J. Huang and M. P. 489 Stryker (2014). "A cortical circuit for gain control by behavioral state." Cell 156(6): 11394901152.

491 Haberly, L. B. (2001). "Parallel-distributed processing in olfactory cortex: new insights

492 from morphological and physiological analysis of neuronal circuitry." Chem Senses 26(5):

$493 \quad 551-576$. 
494 Haberly, L. B. and J. L. Price (1977). "The axonal projection patterns of the mitral and tufted

495 cells of the olfactory bulb in the rat." Brain Res 129(1): 152-157.

496 Haberly, L. B. and J. L. Price (1978). "Association and commissural fiber systems of the

497 olfactory cortex of the rat. II. Systems originating in the olfactory peduncle." L Comp Neurol

498 181(4): 781-807.

499 Igarashi, K. M., N. Ieki, M. An, Y. Yamaguchi, S. Nagayama, K. Kobayakawa, R. Kobayakawa,

500 M. Tanifuji, H. Sakano, W. R. Chen and K. Mori (2012). "Parallel mitral and tufted cell

501 pathways route distinct odor information to different targets in the olfactory cortex." I

502 Neurosci 32(23): 7970-7985.

503 Illig, K. R. (2005). "Projections from orbitofrontal cortex to anterior piriform cortex in the

504 rat suggest a role in olfactory information processing." LComp Neurol 488(2): 224-231.

505 Illig, K. R. and L. B. Haberly (2003). "Odor-evoked activity is spatially distributed in

506 piriform cortex." J Comp Neurol 457(4): 361-373.

507 Jackson, J., I. Ayzenshtat, M. M. Karnani and R. Yuste (2016). "VIP+ interneurons control

508 neocortical activity across brain states." L Neurophysiol 115(6): 3008-3017.

509 Johenning, F. W., P. S. Beed, T. Trimbuch, M. H. Bendels, J. Winterer and D. Schmitz (2009).

510 "Dendritic compartment and neuronal output mode determine pathway-specific long-term

511 potentiation in the piriform cortex." The Journal of Neuroscience 29(43): 13649-13661.

512 Kanter, E. D. and L. B. Haberly (1993). "Associative long-term potentiation in piriform

513 cortex slices requires GABAA blockade." \Neurosci 13(6): 2477-2482.

514 Kanter, E. D., A. Kapur and L. B. Haberly (1996). "A dendritic GABAA-mediated IPSP

515 regulates facilitation of NMDA-mediated responses to burst stimulation of afferent fibers in

516 piriform cortex." LNeurosci 16(1): 307-312.

517 Karnani, M. M., J. Jackson, I. Ayzenshtat, A. Hamzehei Sichani, K. Manoocheri, S. Kim and R.

518 Yuste (2016). "Opening Holes in the Blanket of Inhibition: Localized Lateral Disinhibition

519 by VIP Interneurons." ЦNeurosci 36(12): 3471-3480.

520 Kepecs, A., N. Uchida and Z. F. Mainen (2007). "Rapid and precise control of sniffing during

521 olfactory discrimination in rats." \Neurophysiol 98(1): 205-213.

522 Kuchibhotla, K. V., J. V. Gill, G. W. Lindsay, E. S. Papadoyannis, R. E. Field, T. A. Sten, K. D.

523 Miller and R. C. Froemke (2017). "Parallel processing by cortical inhibition enables context-

524 dependent behavior." Nat Neurosci 20(1): 62-71. 
525 Kumar, A., O. Schiff, E. Barkai, B. W. Mel, A. Poleg-Polsky and J. Schiller (2018). "NMDA

526 spikes mediate amplification of inputs in the rat piriform cortex." Elife 7.

527 Large, A. M., N. A. Kunz, S. L. Mielo and A. M. Oswald (2016). "Inhibition by Somatostatin

528 Interneurons in Olfactory Cortex." Front Neural Circuits 10: 62.

529 Large, A. M., N. W. Vogler, S. Mielo and A. M. Oswald (2016). "Balanced feedforward

530 inhibition and dominant recurrent inhibition in olfactory cortex." Proc Natl Acad Sci U S A

531 113(8): 2276-2281.

532 Lebel, D., Y. Grossman and E. Barkai (2001). "Olfactory learning modifies predisposition for

533 long-term potentiation and long-term depression induction in the rat piriform (olfactory)

534 cortex." Cereb Cortex 11(6): 485-489.

535 Lee, S., J. Hjerling-Leffler, E. Zagha, G. Fishell and B. Rudy (2010). "The largest group of

536 superficial neocortical GABAergic interneurons expresses ionotropic serotonin receptors."

537 The Journal of Neuroscience 30(50): 16796-16808.

538 Lee, S., I. Kruglikov, Z. J. Huang, G. Fishell and B. Rudy (2013). "A disinhibitory circuit

539 mediates motor integration in the somatosensory cortex." Nat Neurosci 16(11): 1662 -

5401670.

541 Li, W., E. Luxenberg, T. Parrish and J. A. Gottfried (2006). "Learning to smell the roses:

542 experience-dependent neural plasticity in human piriform and orbitofrontal cortices."

543 Neuron 52(6): 1097-1108.

544 Linster, C., M. Maloney, M. Patil and M. E. Hasselmo (2003). "Enhanced cholinergic

545 suppression of previously strengthened synapses enables the formation of self-organized

546 representations in olfactory cortex." Neurobiol Learn Mem 80(3): 302-314.

547 Linster, C., B. P. Wyble and M. E. Hasselmo (1999). "Electrical stimulation of the horizontal

548 limb of the diagonal band of broca modulates population EPSPs in piriform cortex." I

549 Neurophysiol 81(6): 2737-2742.

550 Lucas, E. K. and R. L. Clem (2018). "GABAergic interneurons: The orchestra or the

551 conductor in fear learning and memory?" Brain Res Bull 141: 13-19.

552 Luna, V. M. and N. E. Schoppa (2008). "GABAergic circuits control input-spike coupling in

553 the piriform cortex." ЦNeurosci 28(35): 8851-8859.

554 Madisen, L., T. Mao, H. Koch, J. M. Zhuo, A. Berenyi, S. Fujisawa, Y. W. Hsu, A. J. Garcia, 3rd, X.

555 Gu, S. Zanella, J. Kidney, H. Gu, Y. Mao, B. M. Hooks, E. S. Boyden, G. Buzsaki, J. M. Ramirez, A. 
556 R. Jones, K. Svoboda, X. Han, E. E. Turner and H. Zeng (2012). "A toolbox of Cre-dependent

557 optogenetic transgenic mice for light-induced activation and silencing." Nat Neurosci

558 15(5): 793-802.

559 Malenka, R. C. and M. F. Bear (2004). "LTP and LTD: an embarrassment of riches." Neuron

560 44(1): 5-21.

561 Mombaerts, P., F. Wang, C. Dulac, S. K. Chao, A. Nemes, M. Mendelsohn, J. Edmondson and R.

562 Axel (1996). "Visualizing an olfactory sensory map." Cell 87(4): 675-686.

563 Patil, M. M. and M. E. Hasselmo (1999). "Modulation of inhibitory synaptic potentials in the

564 piriform cortex." ЦNeurophysiol 81(5): 2103-2118.

565 Patil, M. M., C. Linster, E. Lubenov and M. E. Hasselmo (1998). "Cholinergic agonist

566 carbachol enables associative long-term potentiation in piriform cortex slices." I

567 Neurophysiol 80(5): 2467-2474.

568 Pfeffer, C. K., M. Xue, M. He, Z. J. Huang and M. Scanziani (2013). "Inhibition of inhibition in

569 visual cortex: the logic of connections between molecularly distinct interneurons." $\underline{\text { Nat }}$

$570 \quad$ Neurosci 16(8): 1068-1076.

571 Pi, H. J., B. Hangya, D. Kvitsiani, J. I. Sanders, Z. J. Huang and A. Kepecs (2013). "Cortical

572 interneurons that specialize in disinhibitory control." Nature 503(7477): 521-524.

573 Poo, C. and J. S. Isaacson (2007). "An early critical period for long-term plasticity and

574 structural modification of sensory synapses in olfactory cortex." I Neurosci 27(28): 7553-

5757558.

576 Poo, C. and J. S. Isaacson (2009). "Odor representations in olfactory cortex: "sparse" coding,

577 global inhibition, and oscillations." Neuron 62(6): 850-861.

578 Poo, C. and J. S. Isaacson (2011). "A major role for intracortical circuits in the strength and

579 tuning of odor-evoked excitation in olfactory cortex." Neuron 72(1): 41-48.

580 Porter, J. T., B. Cauli, K. Tsuzuki, B. Lambolez, J. Rossier and E. Audinat (1999). "Selective

581 excitation of subtypes of neocortical interneurons by nicotinic receptors." The Journal of

582 Neuroscience 19(13): 5228-5235.

583 Pronneke, A., M. Witte, M. Mock and J. F. Staiger (2019). "Neuromodulation Leads to a

584 Burst-Tonic Switch in a Subset of VIP Neurons in Mouse Primary Somatosensory (Barrel)

585 Cortex." Cereb Cortex. 
586 Rennaker, R. L., C. F. Chen, A. M. Ruyle, A. M. Sloan and D. A. Wilson (2007). "Spatial and

587 temporal distribution of odorant-evoked activity in the piriform cortex." J Neurosci 27(7):

588 1534-1542.

589 Saar, D., Y. Grossman and E. Barkai (2002). "Learning-induced enhancement of

590 postsynaptic potentials in pyramidal neurons." 」 Neurophysiol 87(5): 2358-2363.

591 Saar, D., I. Reuveni and E. Barkai (2012). "Mechanisms underlying rule learning-induced

592 enhancement of excitatory and inhibitory synaptic transmission." \Neurophysiol 107(4):

593 1222-1229.

594 Sosulski, D. L., M. L. Bloom, T. Cutforth, R. Axel and S. R. Datta (2011). "Distinct

595 representations of olfactory information in different cortical centres." Nature 472(7342):

596 213-216.

597 Stettler, D. D. and R. Axel (2009). "Representations of odor in the piriform cortex." Neuron

598 63(6): 854-864.

599 Stokes, C. C. and J. S. Isaacson (2010). "From dendrite to soma: dynamic routing of

600 inhibition by complementary interneuron microcircuits in olfactory cortex." Neuron 67(3):

$601452-465$.

602 Strauch, C. and D. Manahan-Vaughan (2018). "In the Piriform Cortex, the Primary Impetus

603 for Information Encoding through Synaptic Plasticity Is Provided by Descending Rather

604 than Ascending Olfactory Inputs." Cereb Cortex 28(2): 764-776.

605 Stripling, J. S., D. K. Patneau and C. A. Gramlich (1988). "Selective long-term potentiation in

606 the pyriform cortex." Brain Res 441(1-2): 281-291.

607 Sturgill, J. F. and J. S. Isaacson (2015). "Somatostatin cells regulate sensory response fidelity

608 via subtractive inhibition in olfactory cortex." Nat Neurosci 18(4): 531-535.

609 Suzuki, N. and J. M. Bekkers (2010a). "Distinctive classes of GABAergic interneurons

610 provide layer-specific phasic inhibition in the anterior piriform cortex." Cereb Cortex

$61120(12): 2971-2984$.

612 Suzuki, N. and J. M. Bekkers (2010b). "Inhibitory neurons in the anterior piriform cortex of

613 the mouse: classification using molecular markers." L Comp Neurol 518(10): 1670-1687.

614 Taniguchi, H., M. He, P. Wu, S. Kim, R. Paik, K. Sugino, D. Kvitsiani, Y. Fu, J. Lu, Y. Lin, G.

615 Miyoshi, Y. Shima, G. Fishell, S. B. Nelson and Z. J. Huang (2011). "A resource of Cre driver 
616 lines for genetic targeting of GABAergic neurons in cerebral cortex." Neuron 71(6): 995-

6171013.

618 Williams, L. E. and A. Holtmaat (2019). "Higher-Order Thalamocortical Inputs Gate Synaptic

619 Long-Term Potentiation via Disinhibition." Neuron 101(1): 91-102 e104.

620 Wilson, D. A. and R. M. Sullivan (2011). "Cortical processing of odor objects." Neuron 72(4):

$621506-519$.

622 Xu, H., H. Y. Jeong, R. Tremblay and B. Rudy (2013). "Neocortical somatostatin-expressing

623 GABAergic interneurons disinhibit the thalamorecipient layer 4." Neuron 77(1): 155-167.

624 Zaborszky, L., J. Carlsen, H. R. Brashear and L. Heimer (1986). "Cholinergic and GABAergic

625 afferents to the olfactory bulb in the rat with special emphasis on the projection neurons in

626 the nucleus of the horizontal limb of the diagonal band." LComp Neurol 243(4): 488-509. 

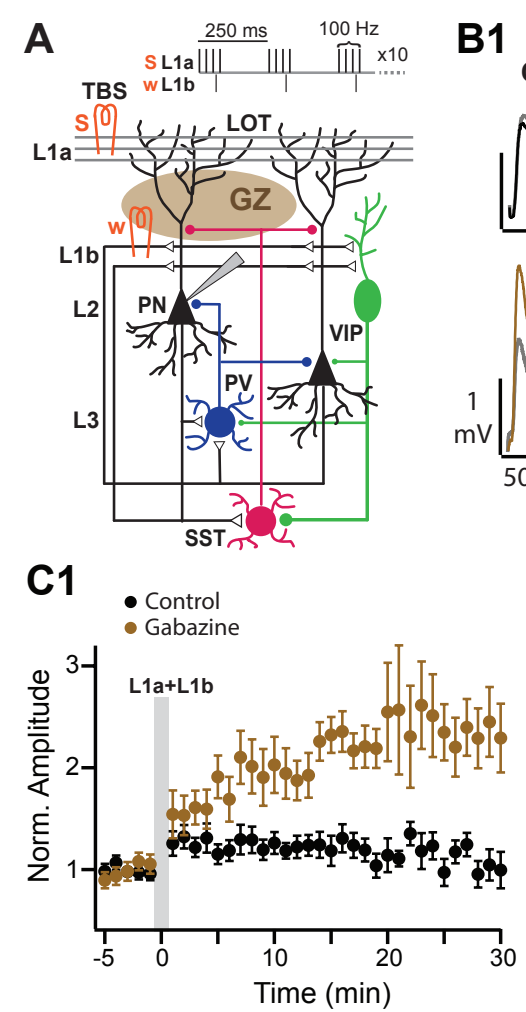

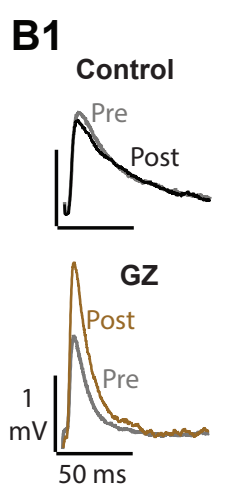

C2

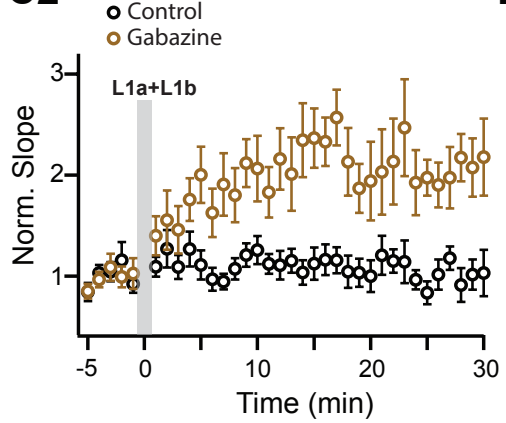

B3
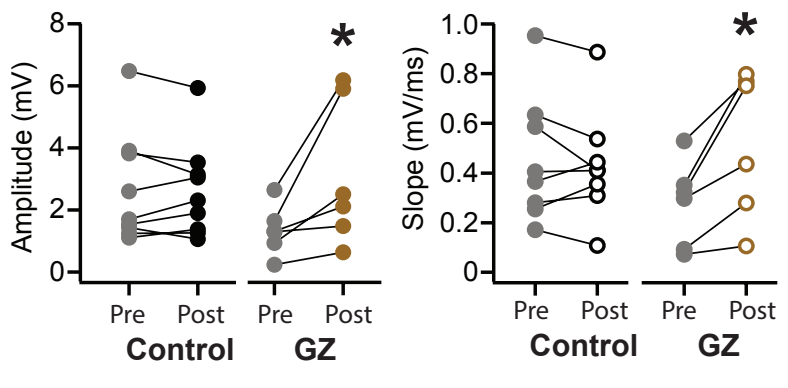

Figure 1: Associative LTP of intracortical synapses is gated by dendritic disinhibition. A) Schematic of APC circuit and stimulation paradigm. Strong (s) TBS of afferents (L1a) is paired with weak (w) single-pulses at the intracortical pathway (L1b). Gabazine (GZ) is focally applied to the dendrite region in L1b (tan oval). B1) Representative average traces pre (gray) and post induction in control (black) and with GZ(Tan). B2) Average EPSP amplitude (mV) pre (-5 to $0 \mathrm{~min}$ ) and post induction (25-30 $\mathrm{min}$ ) with inhibition intact (black) or with dendritic GZ (tan). B3) Average slope ( $\mathrm{mV} / \mathrm{ms})$ of the $20-80 \%$ rising phase of the EPSP pre and post induction. C1) Time course of normalized EPSP amplitude pre and post induction (grey box, $\mathrm{t}=0$ ) in control (black) and with dendritic Gabazine (tan). C2) Time course of normalized EPSP slope, colors as in C2. C3) Normalized EPSP amplitude, and slope 30 min post induction in control (black) and with GZ (tan). 


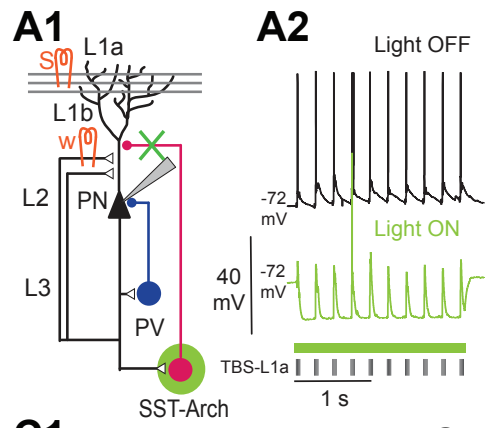

C1

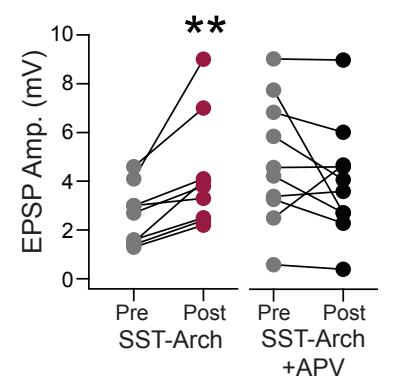

E
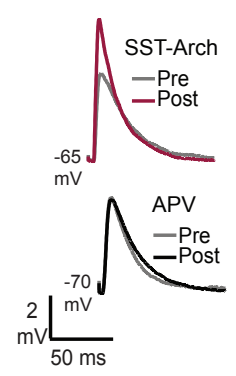

F1

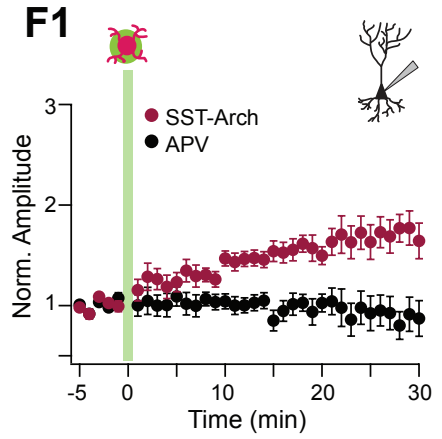

B

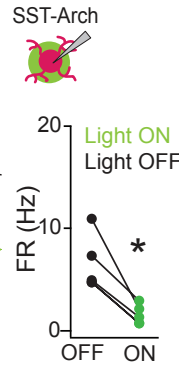

C2
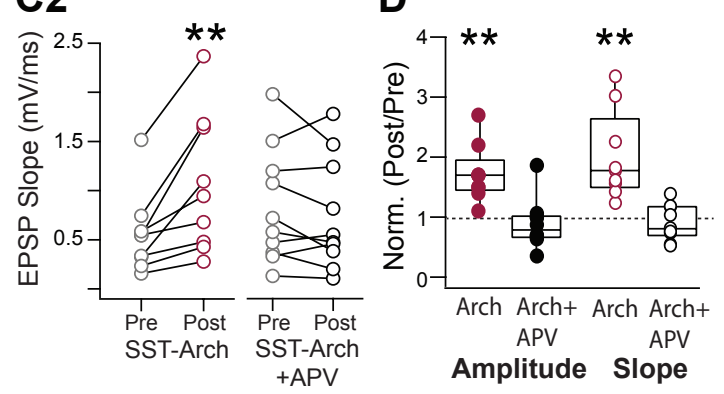

F2

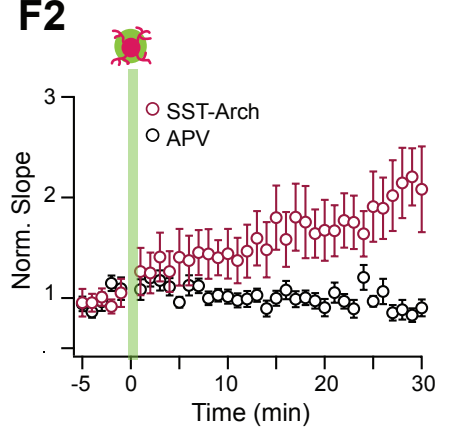

Figure 2: SST-interneuron inactivation promotes associative LTP. A1) Schematic: SST-INs express Archaerhodopsin (Arch). A2) SST-IN spike responses during TBS in control (black) and inactivated (green) conditions ( ${ }^{*} p<0.05$, WSR test). B) PN responses for a single TBS burst. Left : Inactivation of SST-INs enhanced PN depolarization (green vs. black trace). Right: Depolarization during SST-IN inactivation (green) is reduced by APV (gray trace). C1) Average EPSP amplitude $(\mathrm{mV})$ pre (-5 to $0 \mathrm{~min}$ ) and post induction (25-30 min) with SST-inactivation (Magenta) or with SST-inactivation plus bath application of the NMDAR antagonist, APV (black). C2) Average slope $(\mathrm{mV} / \mathrm{ms})$ of the $20-80 \%$ rising phase of the EPSP pre and post induction, colors as in B2. C3) Normalized EPSP amplitude and slope following pairing with SST-IN inactivation (magenta circles). LTP is blocked by bath application APV (black). D1) Representative average traces pre (gray) and post induction in with SST-IN inactivation (magenta) or inactivation plus APV (Black). D2) Time course of normalized EPSP amplitude pre and post induction with inactivation of SST-INs (green box, $t=0$ ) in control (magenta) and with bath application of APV (black). D3) Time course of normalized EPSP slope, colors as in D2. 

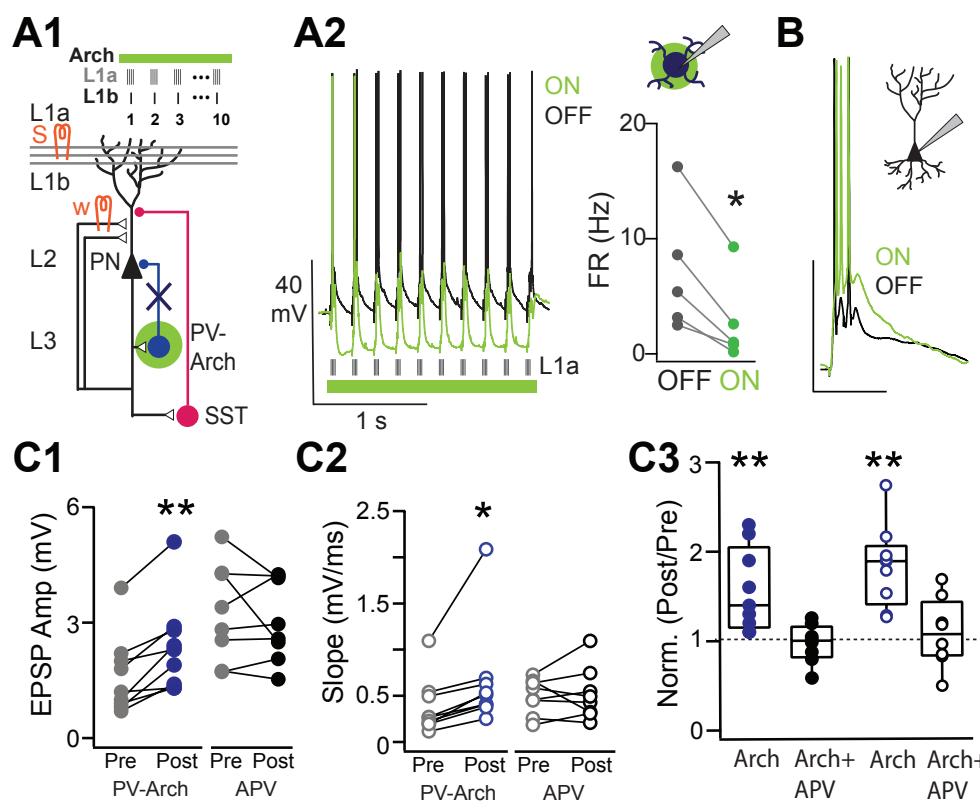

C2

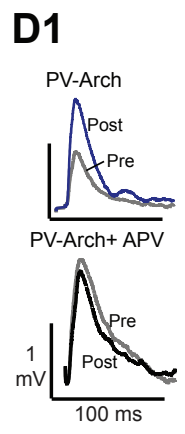

\section{D2}
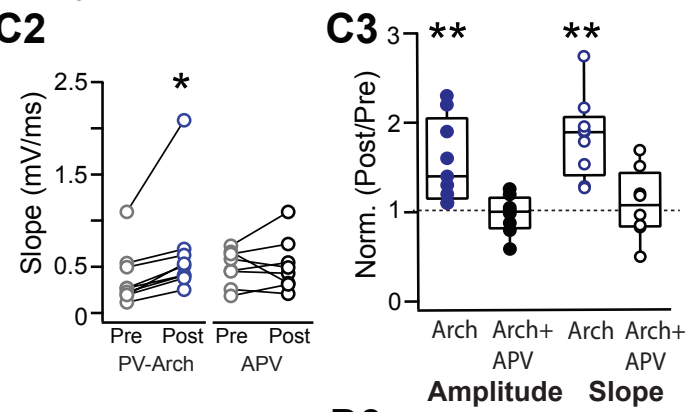

D3
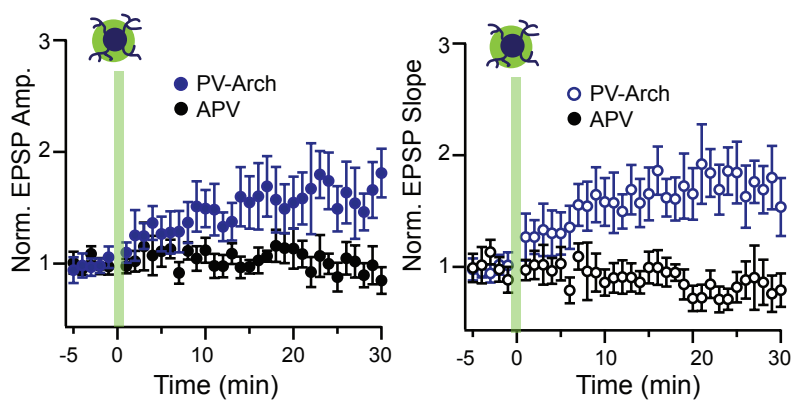

Figure 3: PV-interneuron inactivation during induction promotes associative LTP. A1) Schematic: PV-INs express Archaerhodopsin (Arch). A2) PV-IN spike responses during TBS in control (black) and inactivated (green) conditions. B) Representative PN responses for a single TBS for control (black) versus PV-IN inactivation (green). C1) Average EPSP amplitude $(\mathrm{mV})$ pre (-5 to $0 \mathrm{~min})$ and post induction (25-30 $\mathrm{min})$ with PV-inactivation (Blue) or with PV-inactivation plus bath application of the NMDAR antagonist, APV (black). C2) Average slope ( $\mathrm{mV} / \mathrm{ms})$ of the $20-80 \%$ rising phase of the EPSP pre and post induction, colors as in B2. C3) Normalized EPSP amplitude and slope following pairing with PV-IN inactivation (blue circles) or with inactivation plus bath application APV (black). D1) Representative average traces pre (gray) and post induction in with PV-IN inactivation (blue) or inactivation plus APV (Black). D2) Time course of normalized EPSP amplitude pre and post induction with inactivation of PV-INs (green box, $t=0$ ) in control (blue) and with bath application of APV (black). D3) Time course of normalized EPSP slope, colors as in D2. 


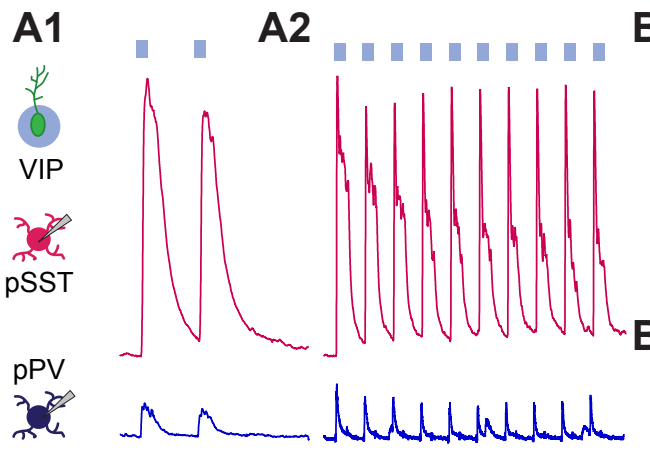

PNA $_{100}^{100}$
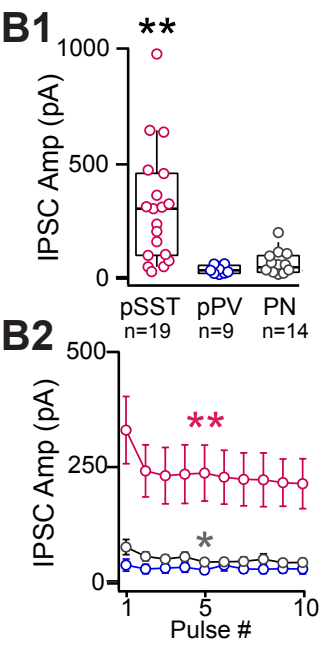
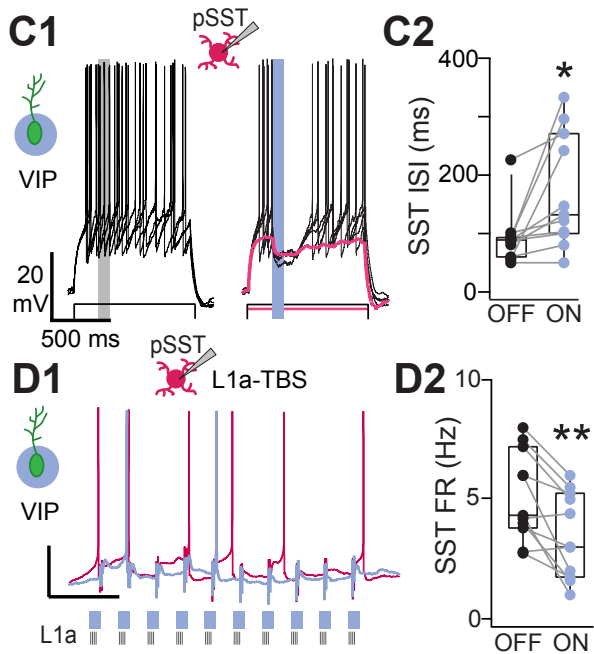

Figure 3: Inhibition by VIP-interneurons in piriform cortex. A1) VIP-INs express ChR2. Optically evoked IPSCs were recorded in putative(p) pSST-INs (magenta), pPV-INs (blue) and PNs (black). A2) Responses of the same neurons (A1) to ten light pulses (100 ms duration, $4 \mathrm{~Hz}$ ). B1) IPSC amplitude was stronger in pSST-INs versus pPV-INs ( $\left.{ }^{*} p: 0.014\right)$ or PNs ( ${ }^{*} p: 0.042$, ANOVA). B2) In pSST-INs, IPSC amplitude diminishes by the 5th pulse of theta stimulation ( ${ }^{* *} \mathrm{p}: 0.009$, paired t-test). C1) IPSCs from VIP-INs delay pSST-IN spike responses during suprathreshold depolarization (4 overlaid traces) Left: Control, Right: activation of VIP-INs (100 ms pulse, blue). Magenta trace: VIP-IN mediated IPSC during subthreshold depolarization. C2) Interspike interval (ISI) was significantly increased during optical activation of VIP-INs (blue circles, $p: 0.016$, paired t-test, $n=11$ ) compared to light off trials (black circles). D1) Spike responses in pSST-INs during TBS in control (magenta trace) and during pulsed light (blue trace). D2) pSST-IN firing rates decreased during TBS on light trials (blue) versus control (black circles, p:0.002, paired t-test, $n=11$ ). 
A1

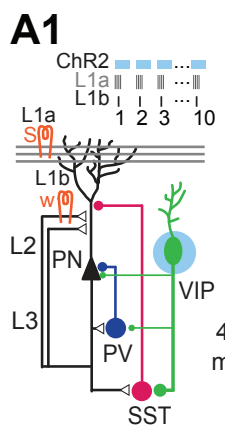

A2

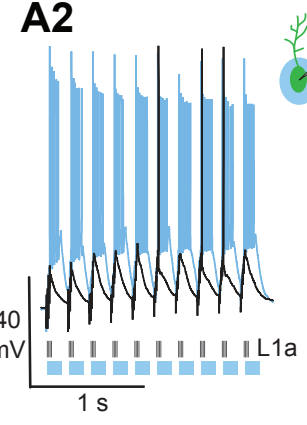

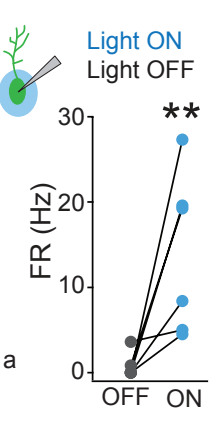

C1

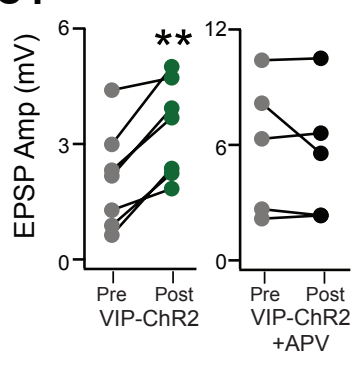

C2

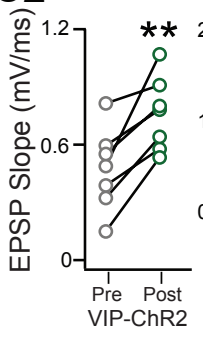

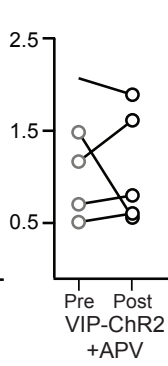
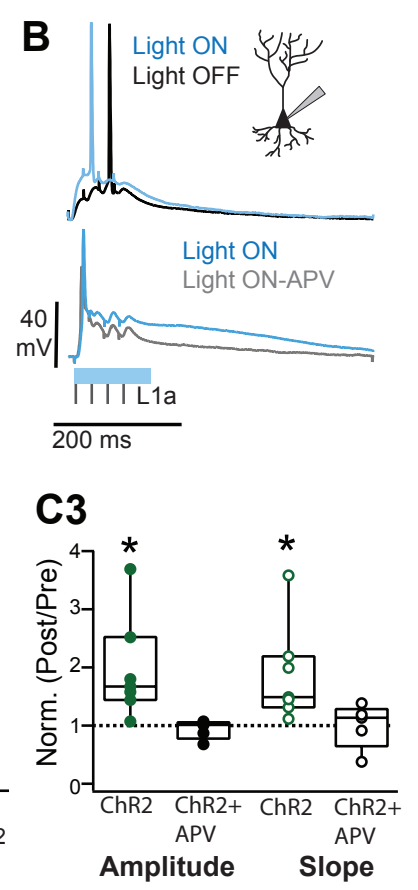

D1
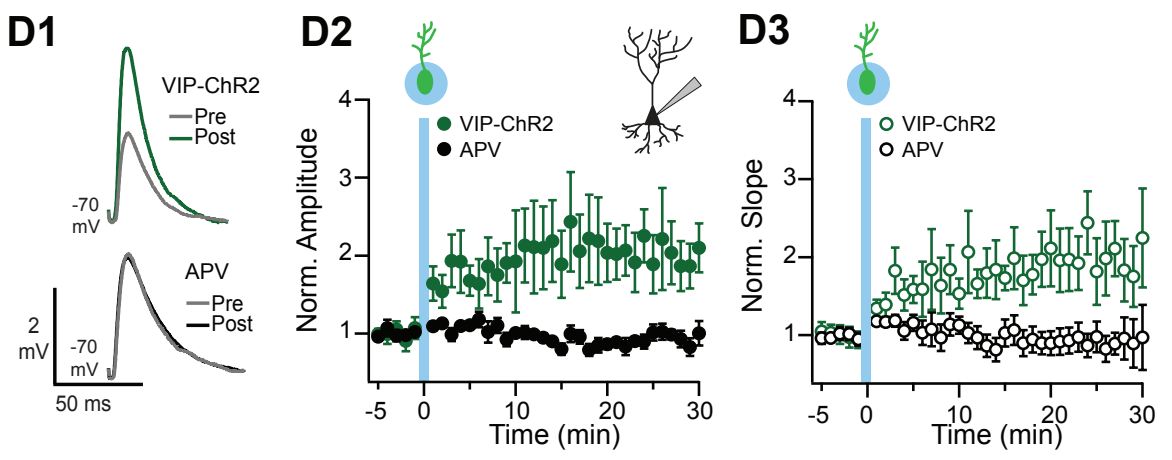

Figure 5: VIP-interneuron activation promotes associative LTP. A1) Circuit schematic: VIP-INs express ChR2 and were activated using theta pulsed light during $L 1 a+L 1 b$ pairing. A2) VIP-IN responses during TBS without (black) and with light (blue). FRs increase during pairing with light (blue circles, $p<0.05$, WSR, $n=7$ ). B) Top: Activation of VIP-INs enhanced PN depolarization during TBS stimulation (blue vs. black trace). Bottom: PN depolarization during VIP-IN activation (blue trace) is reduced by APV (gray trace). C1) Average EPSP amplitude ( $\mathrm{mV}$ ) pre (-5 to $0 \mathrm{~min}$ ) and post induction (25-30 min) with VIP-IN activation (green) or with VIP-IN activation plus bath application of APV (black). C2) Average slope $(\mathrm{mV} / \mathrm{ms})$ of the rising phase of the EPSP pre and post induction, colors as in C1. C3) Normalized EPSP amplitude and slope following pairing with VIP-IN activation (green circles) or with activation plus APV (black). D1) Representative average traces pre (gray) and post induction in with VIP-IN activation (green) or activation plus APV (Black). D2) Time course of normalized EPSP amplitude pre and post induction with activation of VIP-INs (blue box, $t=0$ ) in control (green) and with APV (black). D3) Time course of normalized EPSP slope, colors as in D2. 